\title{
Communication factors affecting the tourism services in the upper northeast of Thailand
}

\author{
Maleerat Ka-kan-dee ${ }^{a^{*}}$ and Sakkarin Nonthapot ${ }^{b}$
}

${ }^{a}$ School of Liberal Arts, Khon Kaen University, Nong Khai Campus, Thailand

${ }^{b}$ Indo-China Country International Trade and Economic Research Sector, Khon Kaen University, Nong Khai Campus

\section{CHRONI CLE}

\section{Article history:}

Received: November 11, 2019

Received in revised format: No-

vember 302019

Accepted: December 18, 2019

Available online:

December 20, 2019

Keywords:

Communication factors

Tourism personnel

Upper Northeast

\section{A B S T R A C T}

The objective of this research was to study the communication factors affecting the tourism services in Loei, Nong Khai, Bueng Kan, Nakhon Phanom, and Mukdahan provinces of Thailand. Purposive sampling was applied, and the sample group comprised 300 accommodation business receptionists and package tour sales staff. A questionnaire was used as a tool to collect the data, and exploratory factor analysis (EFA( and confirmatory factor analysis (CFA( were utilized to analyze the collected data. The findings indicated that the EFA extracted two factors; namely the channel and receiver, which consisted of five variables, and the message and sender, which consisted of five variables that affected the tourism services. Additionally, the results of the CFA showed that the model was consistent with the empirical data ) $\mathrm{APC}=0.90$; Average $\mathrm{R}^{2}=0.80$; $\mathrm{GoF}=0.75$ (. The message and sender affected the tourism services the most, followed by the channel and receiver. Therefore, the relevant agencies and business owners should pay attention to the tourism personnel in terms of message and sender by promoting training and learning to construct understanding in the sent message, be responsible as the sender, and apply the appropriate strategies to send or present the message to the tourism personnel.

\section{Introduction}

\subsection{Background and Significance}

English is one of the most important fundamental languages in the world today. It is an international language and a significant communication tool among the global community. The English language is also a vital gear for communication to propagate and exchange information across various areas and to negotiate businesses. Moreover, using English accurately is a way to build a good relationship between Thai and non-Thai people, to understand the diverse culture of each race, and know how to behave to other cultures accurately and appropriately. Communication in the current world has been developed in terms of technology, so information has been rapidly propagated. One of the key factors affecting this speedy change is communication that generates development in terms of the economy, society, education, culture, etc. This is empirical evidence that communication is a part of human living and society. For humans to constantly function, communication has become a priority and pursued technology and the human way of life, which is a significant mechanism to drive dynamic change toward the future. Tourism services play an important role in the world economy and also generate high income for Thailand from the value adding to the products and services, by increasing more employment opportunities, and distributing income to the rural areas to develop remote regions as natural and man-made tourist sites to attract tourists to visit. Furthermore, tourism and the services business are the key business sectors of Thailand that is now facing tense marketing competition. In Thailand, tourism services are the key source of income for generating foreign revenue, creating jobs, and distributing development to various

* Corresponding author.

E-mail address: maleka@kku.ac.th (M. Ka-kan-dee)

C 2020 by the authors; licensee Growing Science, Canada doi: $10.5267 /$ j.msl.2019.12.024 
regions. Additionally, tourism has a role in the development of the telecommunications infrastructure as well as trading and investment.

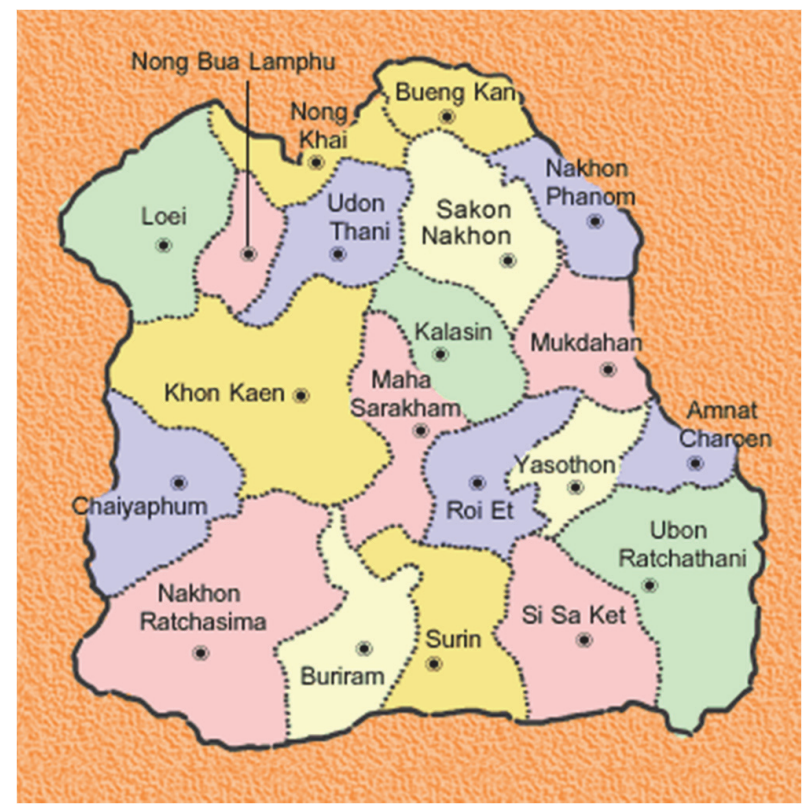

Source: Thailand Traveling Guide (n.d.)

Fig. 1. Map of Upper Northeastern Thailand.

From Fig. 1, it can be seen that Loei, Nong Khai, Bueng Kan, Nakhon Phanom, and Mukdahan provinces are five small adjacent provinces in Northeastern Thailand that are located on the bank of the Khong River opposite the Lao People's Democratic Republic (PDR). They have attractive natural resources, so tourism along the Khong River is filled with charm that fascinates Thai and foreign tourists with the riverine lifestyle of the people, the beautiful nature, folk traditions, and history that bond Thailand-Lao PDR-Vietnam together. These are the assets that have strengthened this area to attract tourists and build tourism services, shops, create investment, and business between countries. Therefore, English is very important as a means of communication among the tourism services personnel, particularly those who have to interact with foreigners. If they are unable to communicate with the tourists, this may cause misunderstanding. Tourism personnel are an important resource in the tourism industry because this group of people has to welcome tourists, but they have a problem communicating with the tourists, as they are not proficient in English. Some of them can speak English moderately, but it is still considered under the communication standard. Sometimes, there are problems caused by misunderstanding and cultural differences between the tourism services personnel and foreign tourists because of the limited English skills. Although Thailand has supportive factors for tourism, the lack of foreign language communication skills is a major barrier (Kasikorn Research Center, 2008).

From the above-mentioned issues, the researcher became aware of the state of the English language communication of the tourism personnel. Moreover, there is a number of research studies about English communication in tourism services in famous tourist cities in Thailand; however, research focusing on the communication factors affecting tourist services in the Upper Northeast of Thailand is limited. Therefore, the researchers investigated the communication factors affecting the tourist services in Loei, Nong Khai, Bung Kan, Nakhon Phanom, and Mukdahan provinces to be a guideline for developing English language skills among the tourism personnel in order to establish a positive image of the Thai tourism industry, create satisfaction among tourists, and enhance the potential and quality of sustainable services.

\section{Relevant Literature}

\subsection{SMCR Concept by Berlo}

Berlo (1960) thoroughly explained the characteristics and factors of the communication process. He summarized that there were six components of communication as follows:

\section{Communication Source 2. Encoder 3. Message 4. Channel 5. Decoder 6. Communication Receiver}

To send and receive the information of the sender and receiver, the communication source and the encoder may be one person called the "source" and the encoder and the receiver is called the "receiver". Thus, the communication model based on Berlo's 
concept consists of the source, message, channel, and receiver. He explained the significant characteristics of each communication component that influenced the success or failure of communication (refer to Fig. 2).

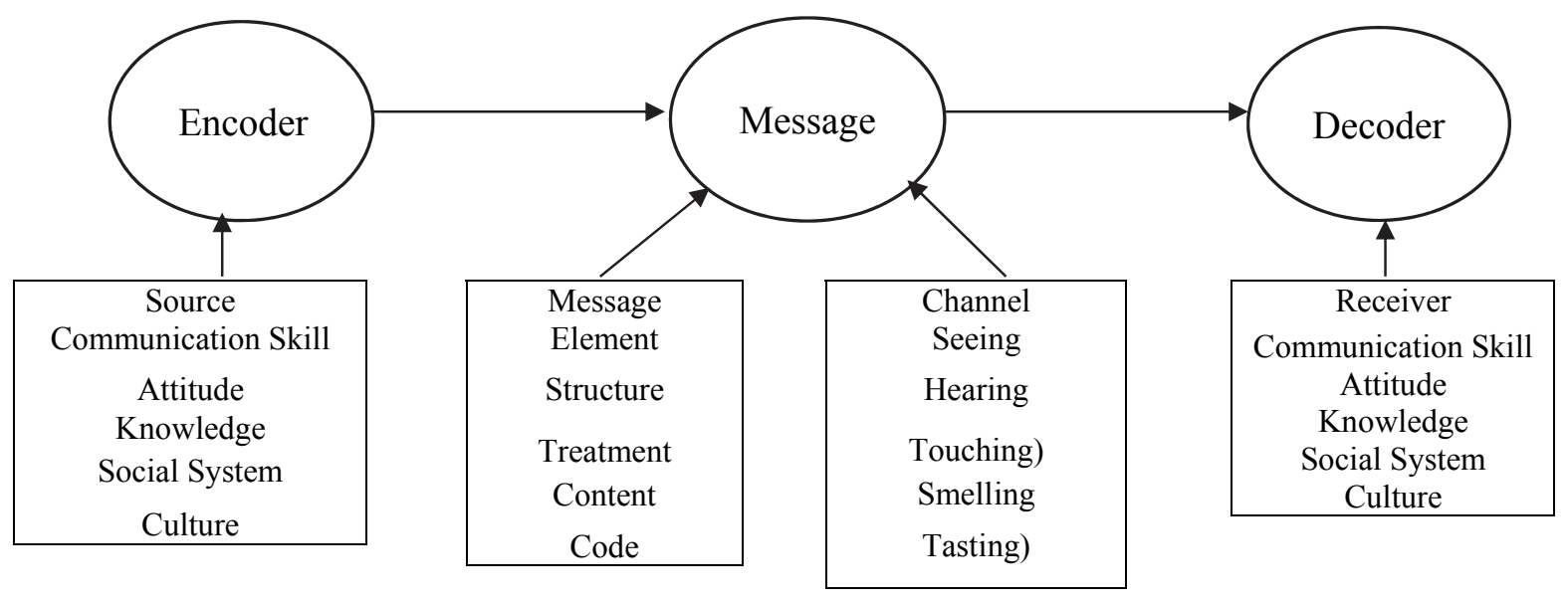

Source: Stead, B. A. (1972).

Fig. 2. Berlo's communication model.

As seen in Fig. 2, Berlo's communication model illustrates the communication components and details of the characteristics of the components as follows:

1) Source - A person who sends or transfers information, idea, emotions, and feelings by encoding it as a signal, symbol and gesture. Berlo believed that communication would be successful if the sender had the characteristics that facilitated the communication success and effectiveness.

2 ) Message - An output of the feelings, emotions and content that the sender would like to convey. It might be in a verbal or non-verbal form.

3) Channel - A means or mediator to send or transfer the message. The channel is the important component of the communication process because it brings the message to the receiver. To send the message to the receiver requires sending it by means of any or all of the five senses, which are hearing, sight, touch, taste, and smell.

4) Receiver - A person who receives the message from the sender. The receiver should have the characteristics of being a good receiver in order to make the communication successful, which includes competency in reading, listening, interpreting, and reading for the main idea. Such competency and proficiency are different in each individual depending on his/her knowledge and experiences. Therefore, the perception would be different.

\subsection{Relevant Research}

To complete the research on the communication factors affecting the tourism services in the Upper Northeast of Thailand, the researchers examined the studies relevant to the communication factors and summarized them. Jaithatkul (2014) studied the organizational communication of the Operation Center of the Salaya Pavilion Hotel and found that the level of opinion on the communication situation relied on the sender and receiver the most. The employees adhered to conducting good behavior and professionalism. Regarding the channel, most communication was formal where the employees were assigned the job according to the hotel's hierarchy. The last one was the message, where the employees used simple spoken language. Furthermore, Sirirat (2015) examined the problems in communication among the personnel in the Central Library of Srinakharinwirot University. The results showed that the sender had the problem the most because of the infrequency of informing information. Sometimes, there was a short notice or delayed notice, so the personnel were unable to perform their duties. Moreover, the message did not reach all personnel since there was no rechecking by the assigned person, and the executive did not transfer the necessary information, as he thought that everyone had noted it. Likewise, the physical structure that was the decentralized work area made the sender and receiver being located in a different location. Others studied the guidelines for the development of organizational communication for Ohgitani (Thailand) Co., Ltd. and found that the level of opinion on the state of communication was on the receiver. The personnel strictly followed the instructions and orders of the supervisor. The second opinion was the sender who worked with the colleagues and other staff. The third opinion was on the message. If the message or the information was in Thai, it would have greater understanding and be clearer than other languages. The last one was the media or channel, which the staff normally used verbal language to communicate. In terms of communication within or between organizations, the sender had the influence on the effectiveness of the operational staff's performance. For external communication of the organization, the receiver, message and channel had no impact on the operational staff's performance. This implied that the operational staff in the Bangkok area who were selected as the sample group had the communication pattern that developed the relationship among the employees in various departments very well. It was likely that they had good skills 
for being the sender, so the communication pattern was effective (Eiamsamang, 2014). From the relevant research on the communication factors, it could be seen that the English communication factors in tourism personnel had never been studied. Therefore, the researcher utilized the communication factors of the employees as the guideline. Jaithatkul (2014) discovered that the employees had the opinion on the sender and receiver the most. Eiamsamran (2014) indicated that the communication within and between organizations had an impact on the effectiveness of the operational staff. Lastly, Sirirat (2015) reported that the problem of organizational communication was caused by the sender the most. Thus, the sender and receiver were the significant factors affecting the communication, which involved the sender, message, channel, and the receiver according to the concept of Berlo (1960).

\section{Research Methodology}

The research on the communication factors affecting the tourism services in the Upper Northeast of Thailand was quantitative study. The research process was as follows:

(1) Studied the concepts, theories and research relevant to the communication factors from documents, articles and research studies that were conducted in Thailand and other countries as the guidelines to create a questionnaire for the tourism personnel about the communication factors affecting the tourism services. Exploratory factor analysis (EFA) was used to analyze the communication components affecting the tourism services and the components were extracted as well as to reduce and classify the variables.

(2) Confirmatory factor analysis (CFA) was used to examine the component structure based on the concept whether it was consistent with the empirical data or not. Then, structural equation modeling (SEM) was used for the analysis.

\subsection{Sample Group}

The sample group comprised 300 accommodation business receptionists and package tour sales staff.

\subsection{Research Tool}

A five-Likert scale questionnaire was used to collect the data from the tourism personnel (Ruengpraphan, 2000: p. 30). The questionnaire asked about the opinion on the communication factors affecting the tourism services in terms of sender, message, channel, and receiver

\subsection{Data Collection}

The data were collected from the 300 sets of questionnaires that were distributed to the sample group.

\subsection{Data Analysis}

The statistics used to analyze the quantitative research were SEM, which included EFA and CFA.

\section{Results}

The study on the communication factors affecting the tourism services in the Upper Northeast of Thailand comprised general information and the communication factors affecting the aforementioned services. The general information of the accommodation business receptionists indicated that from 150 receptionists, 118 receptionists were female $(78.7 \%), 61$ respondents were aged $18-30$ years old (60.7\%), and 64 receptionists had graduated with a bachelor's degree (42.7\%). Regarding the 150 package tour sales staff, 97 of them were female (64.7\%), 61 staff were aged between $31-40$ years old (40.7\%), and 82 respondents had graduated with a bachelor's degree (54.7\%).

The results from the EFA on the communication factors affecting the tourism services showed that KMO $=0.862$ while Bartlett's test of sphericity was 1669.009; $\mathrm{df}=45, \mathrm{p}<.000$. This indicated that the data were appropriate for the EFA (Hair et al., 2010). Then, in examining the Eigenvalues by using the Varimax method, it was found that there were two components that the Eigenvalues were more than 1.00 (refer to Table 1). This implied that the components could be classified into two groups. The first component explained the variance at $51.92 \%$, which was acceptable in the Social Science field whereas the second component explained the variance of the questions at $12.88 \%$. Both components explained the variance of the question at $64.80 \%$ which illustrated that the appropriate component was two components. When comparing all components, the loading should be more than 0.500 . In order to show the classification of the variables, the number of the component variables B1-B5 was arranged with the two components that had the value of $0.71-0.80$ while the component variables B6-B10 were arranged with the component that had the value of 0.66- 0.82 (refer to Table 2 and Fig. 3 ). 
Table 1

Initial Eigenvalues of the accommodation business receptionists and package tour sales staff.

\begin{tabular}{cccc}
\hline \multirow{2}{*}{ Component } & \multicolumn{3}{c}{ Initial Eigenvalues } \\
\cline { 2 - 4 } & Total & \% of Variance & Cumulative \% \\
\hline 1 & 5.19 & 51.92 & 51.92 \\
2 & 1.29 & 12.88 & 64.80 \\
3 & 0.85 & 8.53 & 73.33 \\
4 & 0.76 & 7.61 & 80.94 \\
5 & 0.46 & 4.64 & 85.58 \\
6 & 0.39 & 3.87 & 89.45 \\
7 & 0.33 & 3.30 & 92.75 \\
8 & 0.30 & 2.97 & 95.72 \\
\hline 10 & 0.23 & 2.31 & 98.03 \\
\hline
\end{tabular}

Source: From the calculation.

\section{Table 2}

Rotated component matrix.

\begin{tabular}{|c|c|c|c|}
\hline & \multirow{2}{*}{ Variables } & \multicolumn{2}{|c|}{ Factors } \\
\hline & & 1 & 2 \\
\hline $\mathrm{B} 1$ & Knowledge and competency in customer service. & & 0.74 \\
\hline $\mathrm{B} 2$ & Use of the appropriate strategy to communicate with the customer. & & 0.78 \\
\hline B3 & Ability in using simple and clear words to make the customer easily understand. & & 0.80 \\
\hline B4 & Review and revise the information on the sign before starting the communication. & & 0.77 \\
\hline B5 & $\begin{array}{l}\text { Convey the meaning of the content or information on the notice that is difficult to understand with additional } \\
\text { wording. }\end{array}$ & & 0.71 \\
\hline B6 & The completion of the information about the accommodation and tourism services on the electronic system. & 0.67 & \\
\hline B7 & Sufficient and various communication channels; such as, E-mail and noticeboard. & 0.66 & \\
\hline B8 & Good English listening and speaking skills to perceive and understand the meaning. & 0.81 & \\
\hline B9 & $\begin{array}{l}\text { Perception of the information about the accommodation and tourism services by giving the services based on the } \\
\text { reliability. }\end{array}$ & 0.82 & \\
\hline B10 & Have the ability to perceive the information and accurately transfer knowledge to the customers. & 0.82 & \\
\hline
\end{tabular}

Source: From the calculation.

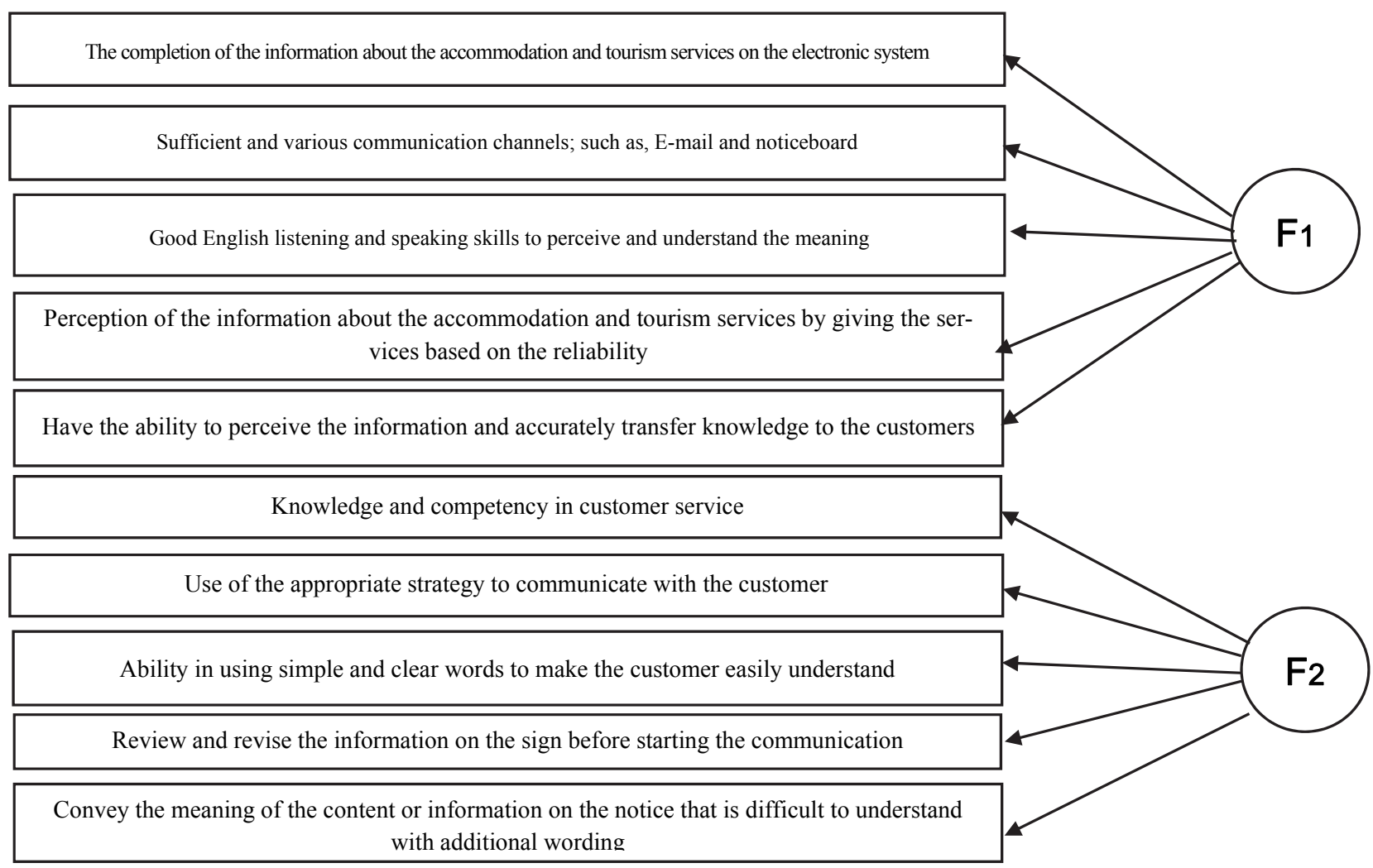

Fig. 3. Results from the EFA 
From Table 2, it was found that Component 1 consisted of the variables B6, B7, B8, B9, and B10 while Component 2 comprised B1, $\mathrm{B} 2$, B3, B4, and B5. When considering the loading of each variable, the value of each variable of all components was higher than 0.600 . This implied that all variables could be classified into any component. To name the component was to reflect the overall meaning of the variables in the group. The component reflected the perspective of the accommodation business receptionists and the package tour sales staff through their opinion on the communication factors. Therefore, each component was renamed as follows:

Component 1 involved five variables, which were the completion of the information about the accommodation and tourism services on the electronic system (B6), the sufficient and various communication channels; such as, E-mail and noticeboard (B7), good English listening and speaking skills to perceive and understand the meaning (B8), perception of the information about the accommodation and tourism services by giving the services based on the reliability (B9), and have the ability to perceive the information and accurately transfer knowledge to the customers (B10). All variables reflected the channel to approach the information about tourism and the properties of the receiver. Therefore, Component 1 was the channel and receiver (F1) (refer to Fig. 3). Component 2 involved five variables, which were knowledge and competency in customer service (B1), the use of the appropriate strategy to communicate with the customer (B2), the ability in using the simple and clear words to make the customer easily understand (B3), review and revise the information on the sign before starting the communication (B4), and convey the meaning of the content or information on the notice that is difficult to understand with additional wording (B5). All variables reflected the state of the capable and knowledgeable person to send a quality message. Therefore, Component 2 was the message and the sender (F2) (refer to Fig. 3). After testing the EFA, this led to the CFA. The initial evaluation results showed that the average path coefficient (APC) was $0.90(\mathrm{P}<0.001)$, the average $\mathrm{R}$-square was $0.80(\mathrm{P}<0.01), \mathrm{GoF}=0.75, \mathrm{SPR}=1.00, \mathrm{RSCR}=1.00$, and SSR $=1.00$. Table 3 shows the appropriateness of the model.

Table 3. Model of the test results appropriateness.

\begin{tabular}{lcll}
\hline \multicolumn{1}{c}{ Values for Evaluation } & Value & \multicolumn{1}{c}{$\begin{array}{c}\text { Significance/ } \\
\text { Evaluation Criteria }\end{array}$} & Evaluation Results \\
\hline $\begin{array}{l}\text { Average path coefficient (APC) } \\
\text { Average R-squared (ARS) }\end{array}$ & 0.90 & $\begin{array}{l}\mathrm{P}<0.001 \\
\mathrm{P}<0.001\end{array}$ & $\begin{array}{l}\text { High level of relationship } \\
\text { High level of relationship }\end{array}$ \\
$\begin{array}{l}\text { Tenenhaus GoF (GoF) } \\
\text { Sympson's paradox ratio (SPR) }\end{array}$ & 0.75 & $\begin{array}{l}\text { Small }>=0.1, \\
\text { medium }>=0.25, \\
\text { large }>=0.36 \\
\text { acceptable if }>=0.7, \\
\text { ideally }=1\end{array}$ & Large \\
acceptable if $>=0.9$, & Accepted \\
ideally $=1$ & acceptable if $>=0.7$ & Accepted \\
R-squared contribution ratio (RSCR) & 1.00 & Accepted \\
Statistical suppression ratio (SSR) & 1.00 & &
\end{tabular}

The appropriateness led to the identification of the reflective model that tested the convergent validity and discriminant validity. The measure with the convergent validity was considered from the indicator loading that was higher than 0.700 with a statistical significance of $99 \%$ of all items (P-value $<0.001$ ). The indicator loading was identified based on the method of Henseler et al. (2009: p. 277-319) (refer Table 4).

\section{Table 4}

The convergent validity of the latent variables with the reflective measure.

\begin{tabular}{|c|c|c|c|}
\hline Construct & Loading & S.E & $\mathrm{P}$-value \\
\hline \multicolumn{4}{|l|}{ Communication factors } \\
\hline 1. CR (Channel and Receiver) & 0.896 & 0.050 & $<0.01$ \\
\hline 2. $\quad$ MS (Message and Sender) & 0.896 & 0.050 & $<0.01$ \\
\hline \multicolumn{4}{|l|}{ CR (Channel and Receiver) } \\
\hline 1. The completion of the information about the accommodation and tourism services on the & 0.752 & 0.051 & $<0.01$ \\
\hline 2. The sufficient and various communication channels; such as, E-mail and noticeboard. & 0.783 & 0.051 & $<0.01$ \\
\hline 3. Good English listening and speaking skills to perceive and understand the meaning. & 0.847 & 0.051 & $<0.01$ \\
\hline 4. Perception of the information about the accommodation and tourism services by giving the & 0.840 & 0.051 & $<0.01$ \\
\hline 5. Ability to perceive the information and accurately transfer knowledge to the customers. & 0.798 & 0.051 & $<0.01$ \\
\hline \multicolumn{4}{|l|}{ MS (Message and Sender) } \\
\hline 1. Competency in customer service. & 0.729 & 0.051 & $<0.01$ \\
\hline 2. The use of the appropriate strategy to communicate with the customer. & 0.751 & 0.051 & $<0.01$ \\
\hline 3. Ability in using simple and clear words to make the customer easily understand. & 0.823 & 0.051 & $<0.01$ \\
\hline 4. Review and revise the information on the sign before starting the communication. & 0.857 & 0.051 & $<0.01$ \\
\hline 5. Convey the meaning of the content or information on the notice that is difficult to understand & 0.847 & 0.051 & $<0.01$ \\
\hline
\end{tabular}

Source: From the calculation.

From Table 4, the factors affecting the communication illustrated how they were defined from both components, the channel and receiver and the message and sender. All loadings were over 0.729 with a reliability of $99 \%$. The discriminant validity of the latent variables in the reflective model and the variables correlation, as well as the reliability of the measure presented the 
composite reliability (CR), average variance extracted (AVE), Cronbach's alpha, $\mathrm{Q}^{2}$, and $\mathrm{R}^{2}$ (refer to Table 5). The minimum CR was 0.900 (should not be lower than 0.600). All AVE were more than 0.600 while all Cronbach's alphas were more than 0.700. $\mathrm{Q}^{2}$ was more than 0 and all $\mathrm{R}^{2}$ were higher than 0.800 (Henseler et al., 2009, p. 277-319). In this research, all $\mathrm{R}^{2}$ were higher than 0.800 . However, to allow for some consideration of the results, the Average $\mathrm{R}^{2}$ should be 0.800 . When considering the results overall, all CR, AVE, Cronbach's alpha, and Q were higher than 0.800, 0.600, 0.700, and 0, respectively. Therefore, this implied that the estimation using the model could predict the internal variables (Chin. 1999, p.8-10). Consequently, the explanation of the estimation with the PLS-SEM was reliable.

Table 5

Statistics showing the discriminant validity.

\begin{tabular}{|c|c|c|c|c|c|}
\hline Construct & $\mathrm{CR}$ & $\mathrm{R}^{2}$ & AVE & Cronbach's Alpha & $\mathrm{Q}^{2}$ \\
\hline CC (Communication) & 0.891 & & 0.803 & 0.754 & \\
\hline CR (Channel and Receiver) & 0.900 & 0.803 & 0.645 & 0.861 & 0.803 \\
\hline MS (Message and Sender) & 0.902 & 0.803 & 0.648 & 0.864 & 0.803 \\
\hline
\end{tabular}

Source: From the calculation.

Table 6 shows the estimation of the cross-construct correlation. It was obvious that the square root of the AVE displayed in the parenthesis was higher than all the AVE values shown in Table 5. Thus, this implied that the measure had the discriminant validity in all constructs. All the square roots of the AVE were higher than 0.800. As a result, the measure of the research had reliable discriminant validity. For this reason, this led to the analysis result as shown in Figure 4 that showed the estimation of the CFA. The correlation of the communication factors affecting the tourism services measured from both communication factors was 0.900 with a significance level of 0.01. In addition, the communication factors affected the tourism services in two aspects, channel and receiver and the message and sender. The message and sender were affected the most $(0.805$ with a significance level of 0.01$)$ followed by the channel and receiver which had the coefficient of 0.803 with a significance level of 0.01 . Likewise, when considering the entire structure, it was found that the empirical data supported all hypotheses or the hypotheses were consistent with the communication factors affecting the tourism services in Loei, Nong Khai, Bung Kan, Nakhon Phanom, and Mukdahan provinces from the two communication factors.

Table 6

Cross construct correlation.

\begin{tabular}{lcc}
\hline Construct & Receiver and Channel & Sender and Message \\
\hline CR (Channel and Receiver) & $(0.803)$ & 0.606 \\
MS (Message and Sender) & 0.606 & $(0.805)$ \\
\hline
\end{tabular}

Source: From the calculation.

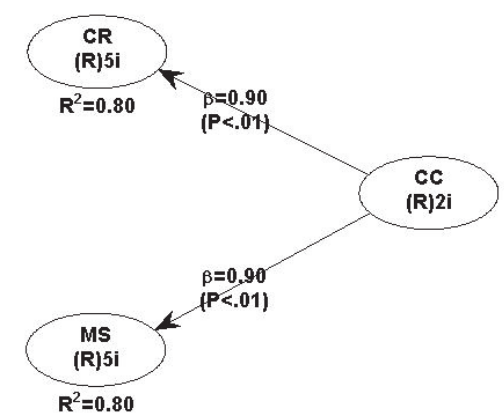

Fig. 4. Result from the CFA

\section{Conclusion and Discussion}

The results of the exploratory factor analysis and confirmatory factor analysis of the communication factors affecting the tourism services have shown that when extracting the communication factors, there were two factors that displayed the Eigenvalues to be more than one, and the accumulated variance was $64.80 \%$. This meant that $64.80 \%$ of the variance between the 10 components could be explained with the components of the two factors. Furthermore, the analysis of the communication factors had variance between the variables at an acceptable level and could be used to create the communication factors model: the channel and receiver and the message and sender, which were consistent with the empirical data. The factor component with the highest weight was message and sender followed by the channel and receiver. When considering the component of each factor, the details were as follows:

(1) The message and sender comprised five components. The first three components that explained the message and sender factor were the use of simple and clear words to make the customer easily understand, the use of the appropriate strategy to 
communicate with the customer, and review and revise the information on the sign before starting the communication. This showed the ability of the sender who chose the appropriate method to communicate with the customer to easily understand and was aware of the appropriateness of the content before starting the communication, which made an impact on the decisionmaking to visit Loei, Nong Khai, Bung Kan, Nakhon Phanom, and Mukdahan provinces. This also conferred with the concept of Satchukorn (2004) who stated that the sender should have a clear intention to let the other person to know his/her objective of sending the message. Moreover, the sender should have the knowledge and understand the message that is being sent, as well as know the preparedness and ability of the receiver by using the appropriate strategy to send or criticize the message.

(2) The channel and receiver comprised five components. The first three components that explained the channel and receiver were the ability to perceive the information and accurately transfer knowledge to the customers, perception of the information about the accommodation and tourism services by giving the services based on the reliability, and good English listening and speaking skills. All these components showed the ability of being the receiver. That was to say the receiver was the person who attempted to learn the information quickly and accurately. Moreover, this was the person who could focus attention on the message from the sender that affected the decision-making to visit Loei, Nong Khai, Bung Kan, Nakhon Phanom, and Mukdahan provinces. This was consistent with the concept of Sa-nguanwongwan (2004, p.115) who gave the definition of communication as a transfer. Therefore, the communication would be successful if it affected the meaning, and the receiver understood it correctly. Communication might be between the individual and/or an organization. From the research results, the relevant agencies and business owners would be able to utilize the information to plan and pilot the guidelines for the development of personnel communication, so they could confidently communicate in the English language with tourists. This would have a positive effect on tourism in the Upper Northeast of Thailand. Furthermore, the business owners and tourism personnel should pay attention to the message and sender factor by promoting training or extra classes that focus on how to become a person who has knowledge and understanding in the message that is being sent, has responsibility as the sender, and uses the appropriate strategy to send the message or present the message to the tourism personnel.

\section{Acknowledgements}

This article is partial research of the "English Language Use in Tourism Services in the Upper Northeast" that has support funding from the National Research Council of Thailand (NRCT) fiscal year 2019.

\section{References}

Berlo, D.K. (1960). The Process of Communication: an Introduction to Theory and Practice.

Chin, W. W. (1998). The partial least squares approach to structural equation modeling. Modern Methods for Business Research, 295(2), 295-336.

Cronbach, L. J. (1990). Essentials of Psychological Testing. Harper Collins: New York.

Eiamsamang, W. (2014). Study on the Internal and External Organizational Communication Pattern Affecting the Effectiveness of Operation Staff Performance in Bangkok. Independent Study of Master of Business Administration Program, Bangkok University.

Hair, J., Black, W. C., Babin, B. J., \& Anderson, R. E. (2010). Multivariate data analysis 7ed. Upper Saddle River.

Henseler, J., Ringle, C. M., \& Sinkovics, R. R. (2009). The use of partial least squares path modeling in international marketing. In New challenges to international marketing (pp. 277-319). Emerald Group Publishing Limited.

Jaithatkul, W. (2014). Organizational Communication the Operation Center of The Salaya Pavilion Hotel. Academic Service Journal, Prince of Songkla University. Year $25^{\text {th }}, 3$ Edition.

Kasikorn Research Center. (2008). Intense Competition among Language Institutes for AEC. Watch the Leading for the Market Share. https://www.kasikornresearch.com. Retrieved on 9 October 2019.

Rovinelli, R. J., \& Hambleton, R. K. (1977). On the use of content specialists in the assessment of criterion-referenced test item validity. Dutch Journal of Educational Research, 2, 49-60.

Ruengpraphan, C. (2000). Fundamental of Statistics, $5^{\text {th }}$ ed., Khon Kaen University.

Sa-nguanwongwan, W. (2004). Organizational Management and behavior. $2^{\text {nd }}$ Edition. Bangkok: Odeon Store.

Satchukorn, S. (2004). Training Techniques. Bangkok: Sai Tharn.

Sirirat, J. (2015). Problem about Communication of the Personnel in Organization: A Case study of the Library Center of Srinakharinwirot University. Srinakharinwirot University.

Stead, B. A. (1972). Berlo's communication process model as applied to the behavioral theories of Maslow, Herzberg, and McGregor. Academy of Management Journal, 15(3), 389-394.

Thailand Traveling Guide. (n.d.). Exploring Isan: Thailand's Frontier. http://thairoam. blogspot.com/p/exploring-isan-thailands-frontier.html Retrieved on 8 January 2019.

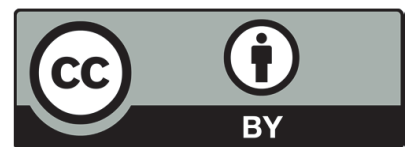

(C) 2020 by the authors; licensee Growing Science, Canada. This is an open access article distributed under the terms and conditions of the Creative Commons Attribution (CC-BY) license (http://creativecommons.org/licenses/by/4.0/). 